\title{
Sandpile model on an optimized scale-free network on Euclidean space
}

\author{
R. Karmakar and S. S. Manna \\ Satyendra Nath Bose National Centre for Basic Sciences Block-JD, Sector-III, Salt Lake, Kolkata-700098, India
}

\begin{abstract}
Deterministic sandpile models are studied on a cost optimized Barabási-Albert (BA) scale-free network whose nodes are the sites of a square lattice. For the optimized BA network, the sandpile model has the same critical behaviour as the BTW sandpile, whereas for the un-optimized BA network the critical behaviour is mean-field like.

PACS numbers: 05.65.+b 05.70.Jk, 45.70.Ht 05.45.Df
\end{abstract}

Various models of Statistical Mechanics which are usually studied on regular lattices are being studied in recent years on graphs or networks of very complex structures. For example, the Ising model has been studied both on the Small-World Networks (SWN) 1, 2] and on ScaleFree Networks (SFN) [3]. The phenomenon of Percolation has also been studied on such networks [4, [5]. In addition disease spreading models like susceptible-infectedsusceptible (SIS) [6] have been studied on networks for spreading diseases in the society or spreading of viruses in the Internet.

Over the last few years it has been observed that the nodal degree distributions of many real-world networks, e.g., World Wide Web [7] and the Internet [8] are characterized by power law tails: $P(k) \sim k^{-\gamma}$ (degree $k$ of a node being the number of links attached to it). These networks are called 'scale-free networks' 9, 10, 11, 12] due to the absence of a characteristic value for nodal degrees. Theoretically a number of graphs are generated to model SFNs. One of them is by Barabási and Albert (BA) which has the following ingredients, namely: (i) A network grows from an initial set of $m_{o}$ nodes with links connecting all $m_{o}$ nodes. At every time step a new node is introduced and is randomly linked to $m\left(<m_{o}\right)$ distinct previous nodes. (ii) Any of these $m$ links introduced at time $t$ connects a previous node $i$ with an attachment probability $\pi_{i}(t)$ which is linearly proportional to the degree $k_{i}(t)$ of the $i$-th node at time $t: \pi_{i}(t) \propto k_{i}(t)$. For BA model $\gamma=3[9]$.

Sandpile models are the prototype models of Selforganized Criticality (SOC) 13, 14, 15, 16, 17. In these models, long ranged correlations both in space as well as in time spontaneously emerge under a self-organizing dynamics, in absence of a fine tuning parameter. In its very general form, a sandpile model can be defined on an arbitrary connected graph, having a set of vertices connected by another set of edges. An integer height variable $h_{i}$ representing the number of grains in the sand column is associated with every vertex $i$ of the graph. Starting from an arbitrary initial sand height distribution the system is driven by adding unit grains of sand at the randomly selected vertices $h_{i} \rightarrow h_{i}+1$. This sand column is said to be unstable when the height $h_{i}$ exceeds a pre-assigned threshold value $h_{c}$. An unstable sand column must topple and in a toppling it looses some grains which are distributed among the neighboring sites 13. This creates an avalanche of sand column topplings and the strength of such activity measures the size of the avalanche. There must be some 'sinks', i.e., a set of vertices through which grains flow out of the system so that in the steady state the balance of fluxes of inflow and outflow currents is maintained.

In this paper, we have studied the deterministic sandpile model on a scale-free network placed on an Euclidean substrate, namely a square lattice. The motivation of this study is to acquire support for the validity of our recent conjecture [18] that in a sandpile model the precise balance at all lattice sites (except on the boundary) between the number of outflowing grains $H_{i}$ which are distributed among the neighbouring sites in a toppling at the site $i$ and the number of inflowing grains $H_{i}^{\prime}$ received by the site $i$ when its all neighbouring sites topple once ensures that the sandpile model behaves like the BTW model 13. with a multiscaling avalanche size distribution. The absence of the site-to-site balance of $H_{i}=H_{i}^{\prime}$ leads to the behaviour of Manna sandpile [14]. Below we define and study the sandpile model on the SFN where $H_{i}$ is equal to the degree $k_{i}$ of the SFN and therefore is an extremely fluctuating quantity. In spite of that the equality $H_{i}=H_{i}^{\prime}$ is maintained by construction. We see below that the sandpile model on the optimized SFN indeed behaves like the BTW model.

Recently, BTW sandpile model has been studied on a static model of SFN [19]. In contrast to the usual sandpile models there are no specific sinks at fixed positions. Instead, during a toppling any grain can evaporate from the system from any arbitrary node with a small probability $f$. The distribution of avalanche sizes $(s)$ which do not dissipate (i.e., grains do not evaporate in these avalanches) is:

$$
\operatorname{Prob}(s) \sim s^{-\tau} \exp \left(-s / s_{c}\right)
$$

where the cut-off of the avalanche size $s_{c} \sim 1 / f$. It is to be noted that the cut-off size does not depend on the network size $N$ but only on the dissipation rate.

To claim that a dynamical process active in a system is self-organized critical, it is important to ensure that both long ranged spatial and temporal correlations dynamically evolve in this system. For the ordinary BTW or 


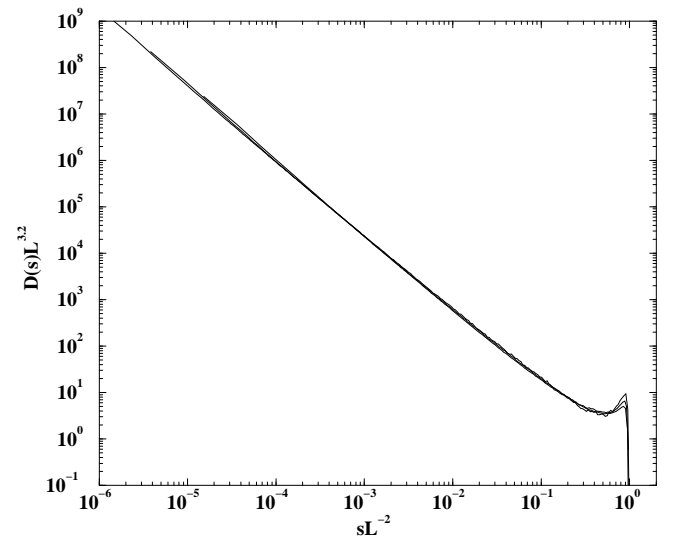

FIG. 1: Scaling of the probability distribution of avalanche sizes for the BTW model on the un-optimized SFN for different system sizes: $L=256,512$ and 1024 . The data collapse gives the values of the scaling exponents $D=2$ and $\beta=3.2$ giving the avalanche size exponent $\tau_{u n-o p t}=1.6$.

Manna sandpiles on systems of spatial extension $L$ this is verified in the following ways: (i) The avalanche size distribution has a power law distribution $\operatorname{Prob}(s, L) \sim s^{-\tau}$ for some intermediate range and this range should increase with the system size as the cut-off of the avalanche size distribution increases as $s_{c}(L) \sim L^{D}$. (ii) The average size of the avalanches increases with the system size $L,\langle s(L)\rangle \sim L^{\nu}$ and $\nu=2$ since the grains while executing a diffusive motion have to travel distances of the order of $L$ to go out of the system through sinks situated on the boundary Non-zero values of $\nu$ and $D$ indicate that system has avalanches of all length scales and the process is indeed critical.

If a sandpile is grown in a closed system (i.e., a system which has no sinks and grains do not evaporate from this system) the system eventually reaches a state when an "infinite avalanche" which continues for ever and never stops. Now, if a slow dissipation rate is introduced like every $1 / f$ topplings one grain is dissipated from any arbitrary site of the system, there is no infinite avalanche, the system indeed reaches a stationary state, but the avalanche sizes are no more of all length scales. This is because the large avalanches loose their strengths by dissipation of grains.

For a network or a graph in general, there is no concept of space, only the connections by links between the nodes. One still can define a distance between an arbitrary pair of nodes on a network measured by the number of links on the shortest path connecting the two points. The largest of all possible shortest paths is called the diameter of the network. A small world network has the diameter varying logarithmically with the number of nodes: $\mathcal{D}(N) \propto \log N$. Since scale-free networks are small world networks, it is difficult to observe long ranged spatial correlations in sandpile model on SFNs.

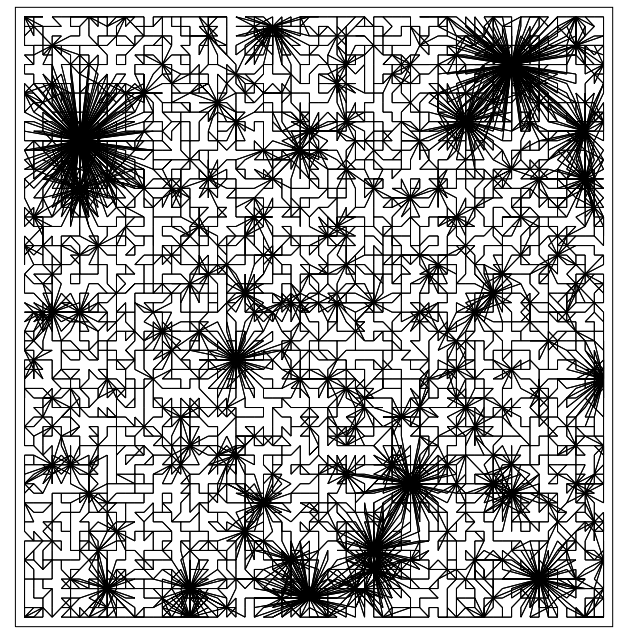

FIG. 2: A cost optimized Barabási-Albert scale-free network on a square lattice of size $L=64$. The SFN of $N=L^{2}$ nodes is generated by usual BA algorithm whose nodes are randomly assigned lattice sites. The cost function $\mathcal{C}$ (the total wiring length) is then minimized by a large number of trials as described in the text keeping the nodal degree distribution intact. Large degree nodes are visible.

Here we study the sandpile model on a SFN constructed on a square lattice of size $L \times L$. We first construct a BA SFN of $N=L^{2}$ nodes. The network starts growing with an initial set of $m_{o}=(m+1)$ nodes. Each of these nodes is linked to all other $m$ nodes forming a $(m+1)$-clique. After that new nodes are added to the network one by one and each such node is connected to $m$ randomly selected distinct nodes of the already grown network with probability $\pi_{i}(t)$. This process stops when the network size has grown to $L^{2}$ nodes. In our calculation we use $m=2$, therefore our network has $L^{2}$ nodes, $2 L^{2}-3$ links among the nodes and has many loops. The nodes of the network are then assigned randomly with uniform probability the sites of the square lattice. If two nodes are linked, the corresponding lattice sites are connected by straight lines. Thus we place the BA SFN on the square lattice.

Clearly the degree distribution of such an Euclidean SFN is exactly the same as that of the BA SFN. To study the sandpile model we assume that each site (except for sites on the boundary) has a site dependent critical height $h_{i}^{c}$ of stability which is equal to the degree $k_{i}$ of the node at that site. Therefore in a toppling, the sand height at site $i$ is reduced to: $h_{i} \rightarrow h_{i}-h_{i}^{c}$ and in a deterministic toppling dynamics like BTW model, all the $k_{i}$ neighbours receive one grain each. The outlet of the system is at the boundary. Therefore every boundary site (except the corner sites) has the threshold heights $h_{i}^{c}=k_{i}+1$. This implies that in a toppling at the boundary site one grain goes out of the system and never comes back. Similarly 


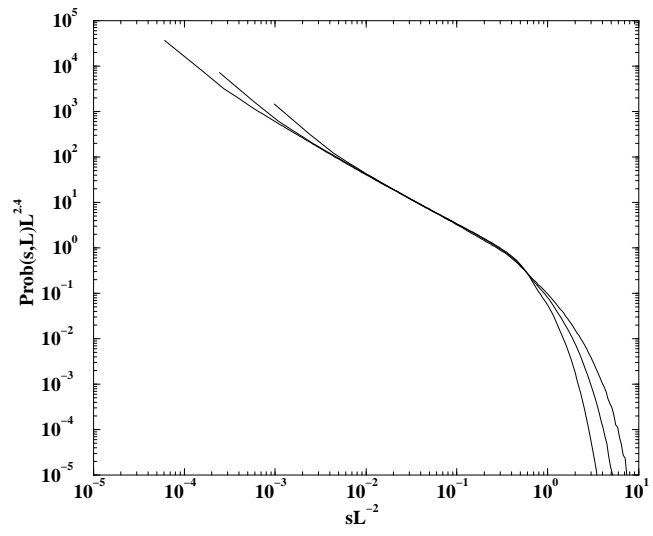

FIG. 3: Scaling of data for the probability distribution of avalanche sizes for the BTW model on optimized SFN and for different system sizes: $L=32,64$ and 128. The data collapse gives the values of the scaling exponents $D=2$ and $\beta=2.4$ giving the avalanche size exponent $\tau_{\text {opt }} \approx 1.2$.

at the corner sites the threshold heights are $h_{i}^{c}=k_{i}+2$. Such mechanism of outflow of grains through the boundary sites guarantees that the sandpile dynamics on the Euclidean SFN must reach a stationary state.

In this Euclidean SFN any site is connected to any other site with equal probability and therefore the average link length $\left\langle\ell_{i j}\right\rangle$ is large and of the order of the system size $L$. In a toppling the grains therefore jump large distances on the average. We first study a deterministic sandpile model on such a network. In this sandpile model a grain jumps a distance around $L$ in a toppling. Consequently, the spatial extent of all avalanches, small or big, are around $L$.

The avalanche size is measured in two ways: (i) the total number of topplings $s$ and the number of distinct sites toppled $a$. A power law distribution of the avalanche sizes with a simple finite size scaling implies that the distribution function $\operatorname{Prob}(s, L)$ obeys the following scaling form:

$$
\operatorname{Prob}(s, L) \sim L^{-\beta} f\left(\frac{s}{L^{D}}\right),
$$

where the scaling function $f(x) \sim x^{-\tau}$ in the limit of $x \rightarrow 0$ giving $\tau=\beta / D, \tau$ and $D$ are the exponents of the avalanche size distribution. One immediate way to check validity of Eqn. (1) is to attempt a data collapse by plotting $L^{\beta}$ Prob vs. $s / L^{D}$ with trial values of the exponents. It is now well known in the literature that for the Manna stochastic sandpile model the distribution obeys FSS with $\tau_{\text {Manna }} \approx 1.28$ where as for the BTW sandpile the probability distribution $\operatorname{Prob}(s, L)$ of this measure has been found recently to obey a multiscaling ansatz 22, 23]. In Fig. 1 we plot the scaling of the avalanche size distribution data for the BTW sandpile on the un-optimized BA SFN on the square lattice for $L=256,512$ and 1024. The best collapse works
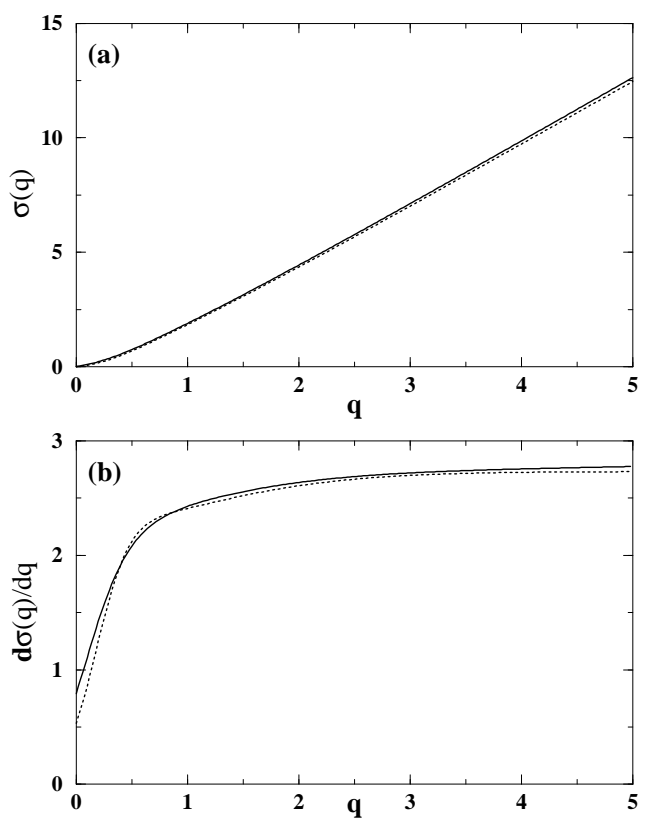

FIG. 4: Comparison of the BTW model on ordinary square lattice (solid line) and the BTW model on the optimized SFN on square lattice (dotted line): (a) the moment exponents $\sigma(q)$ vs. $q$ and (b) $d \sigma(q) / d q$ vs. $q$.

for $D=2$ and $\beta=3.2$, giving $\tau_{\text {un-opt }}=\beta / D=1.6$. The stochastic Manna sandpile is also studied on the unoptimized BA SFN on the square lattice for system sizes upto $L=1024$ again. We estimated $D=2$ and $\beta=3.0$ giving $\tau_{u n_{o} p t}=1.5$. We believe that $\tau_{u n-o p t}$ for both the BTW and Manna sandpiles on the un-optimized BA SFNs are indeed mean-field like and both the exponents should be actually 1.5. Similar slight deviation from 1.5 was also observed in [19] for the deterministic case. It is also observed that the area $a$ has a similar distribution.

Recently a cost optimized SFN on Euclidean space has been constructed [20] where the total sum of the link lengths is optimized keeping the nodal degree distribution exactly same as that of the original SFN. For such a construction one defines a cost function $\mathcal{C}(N)$ as the total wiring length in terms of the symmetric adjacency matrix $\mathbf{A}$ of size $N \times N$ (which has elements $a_{i j}=1$ if there is a link between the pair of nodes $i$ and $j$ and 0 otherwise) and the distance $\ell_{i j}$ between nodes $i$ and $j$ as $\mathcal{C}(N)=\Sigma_{i>j} a_{i j} \ell_{i j}$. The optimization process is essentially a rewiring process maintaining the nodal degree distribution intact. It starts with a BA SFN constructed on a square lattice as mentioned above. A pair of distinct links of the SFN is chosen whose nodes are not linked otherwise. One end of each link is then opened and rewired suitably to another node of the quartet so that total sum of the rewired length is smaller. More precisely, the first node $n_{1}$ is randomly selected from the set of $N$ nodes and the second node $n_{2}$ is randomly selected from the $k_{1}$ 
neighbours of $n_{1}$. In the same way $n_{3}\left(\neq n_{1} \neq n_{2}\right)$ is selected randomly from $N$ nodes and $n_{4}\left(\neq n_{1} \neq n_{2}\right)$ is chosen from $k_{3}$ neighbours of $n_{3}$. Clearly this move conserves the link numbers as well as degree distribution. Rewiring is done following this decision: If both $n_{1} n_{3}$ and $n_{2} n_{4}$ are not linked and also $\ell_{12}+\ell_{34}$ is greater than $\ell_{13}+\ell_{24}$ we link $n_{1} n_{3}$ and $n_{2} n_{4}$. Another possibility is if $n_{1} n_{4}$ and $n_{2} n_{3}$ are not linked but $\ell_{12}+\ell_{34}$ is greater than $\ell_{14}+\ell_{23}$ then we link $n_{1} n_{4}$ and $n_{2} n_{3}$. If both cases are possible we accept one of them with probability $1 / 2$. If only one is satisfied we accept that. After rewiring we remove the links $n_{1} n_{2}$ and $n_{2} n_{4}$. If none of the two is satisfied we go for a fresh trial. On repeated trials of these moves the cost function gradually decreases. Initially it decreases very fast but eventually the success rate becomes very slow. To monitor the optimization process we kept track of the average link length. Our best possible effort yields the average link length $\left\langle\ell_{i j}\right\rangle \approx 1.75$ lattice constant. A picture of the optimized network is given in Fig. 2. In this best possible optimized network the link lenghs $\ell$ have an exponential distribution as: $\mathcal{D}(\ell) \sim \exp (-g \ell)$ with $g \approx 1.16$. Also the diameter of the network $\mathcal{D}(N)$ is measured and is observed to grow as $N^{\mu}$ where $\mu$ is estimated to be $0.40 \pm 0.02$. Therefore this network is scale-free but not a small-world network.

The deterministic BTW sandpile model is then studied on such a network. The avalanche size distribution is calculated for three different system sizes $L=32,64$ and 128. It was difficult to go beyond this size because of the large optimization times required. First we tried to make a scaling plot of the size distribution data. In Fig. 3 we show this plot, which shows reasonably well collapse of the data in the intermediate range of the avalanche sizes. The corresponding $\beta$ and $D$ values fitted are 2.4 and 2 respectively giving a possible value of $\tau_{\text {opt }} \approx 1.2$. However for large avalanche sizes the collapse is much worse and the data for different system sizes separate out. This is a typical behaviour of the BTW like models which show strong presence of the multiscaling behaviour 22, 23].

The mutiscaling behaviour is studied in more detail by the evaluation of the various moments of the avalanche size probability distribution. The $q$-th moment of the distribution is defined as $\left\langle s^{q}\right\rangle=\int s^{q} \operatorname{Prob}(s, L) d s$. In case the distribution $\operatorname{Prob}(s, L)$ obeys the finite size scaling behaviour for the whole range of avalanche sizes, it can be shown that $\left\langle s^{q}\right\rangle \sim L^{\sigma(q)}$ where $\sigma(q)=D(q-\tau+1)$ for $q>\tau-1$ and $\sigma(q)=0$ for $0<q<\tau-1$. The $q$ dependent exponent $\sigma(q)$ is determined from the slope of the plot of $\log \left\langle s^{q}(L)\right\rangle$ with $\log L$, which in our case are for $L=32,64$ and 128. The interval between successive $q$ values is 0.02 and moments are calculated at 251 values of $q$ between 0 and 5. In Fig. 4(a) we show a plot of $\sigma(q)$ vs. $q$ on a linear scale. In Fig. 4(b) the derivative of $\sigma(q)$ is plotted with $q$. Had the $\operatorname{Prob}(s, L)$ followed a simple FSS behaviour the $d \sigma(q) / d q$ in Fig. 4(b) would have saturated for large $q$ values. In stead, the curve gradually increases with $q$, very similar to the multiscaling behaviour of BTW model. To compare we plot both $\sigma(q)$ and $d \sigma(q) / d q$ of the ordinary BTW on square lattice studied for same system sizes with different line styles. We see that in both plots the behaviour is very similar and the difference between the two curves is very small, within 2-3\%.

The stochastic Manna sandpile is also studied on the optimized SFNs for small system sizes $L 32,64$ and 128 . The scaling exponents are estimated as: $D=2.62$ and $\beta=3.4$ giving $\tau_{o p t} \approx 1.3$. This value of $\tau$ is compared with the corresponding $\tau \approx 1.28$ value of the ordinary Manna sandpile.

To summarize, we studied the BTW sandpile model on a Barabási-Albert scale-free network of $N=L^{2}$ nodes where the nodes are the sites of a square lattice of size $L$. The SFN is then optimized minimizing the total wiring length but keeping the degree distribution intact. On such an optimized SFN on the Euclidean space we observe that the sandpile model has the same scaling behaviour as the BTW model where as the deterministic sandpile on the un-optimized SFN has a mean-field like behaviour.

[1] A. Pekalski, Phys. Rev. E, 64, 057104 (2001).

[2] C. P. Herrero, Phys. Rev. E, 65, 066110 (2002).

[3] A. Aleksiejuk, J. A. Holyst, and D. Stauffer, Physica A 310, 260 (2002).

[4] M. E. J. Newman, D. J. Watts, Phys. Rev. E 60, 7332 (1999).

[5] N. Schwartz, R. Cohen, D. ben-Avraham, A.-L. Barabasi, S. Havlin, Phys. Rev. E 66, 015104 (2002).

[6] M. Barthelemy, A. Barrat, R. Pastor-Satorras, A. Vespignani, arXiv:cond-mat/0410330

[7] S. Lawrence and C. L. Giles, Science, 280, 98 (1998); Nature, 400, 107 (1999), R. Albert, H. Jeong and A.-L. Barabási, Nature, 401, 130 (1999).

[8] M. Faloutsos, P. Faloutsos and C. Faloutsos, Proc. ACM SIGCOMM, Comput. Commun. Rev., 29, 251 (1999).

[9] A.-L. Barabási and R. Albert, Science, 286, 509 (1999); R. Albert and A.-L. Barabási, Rev. Mod. Phys. 74, 47 (2002).

[10] A.-L. Barabási, Linked: The New Science of Networks, Perseus Publishing, 2002.

[11] S. N. Dorogovtsev and J. F. F. Mendes, Evolution of Networks, Oxford University Press, 2003; M. E. J. Newman, SIAM Review 45, 167 (2003).

[12] A. Vazquez, R. Pastor-Satorras and A. Vespignani, cond-mat/0206084

[13] P. Bak, C. Tang and K. Wiesenfeld, Phys. Rev. Lett. 59, 381 (1987); P. Bak, C. Tang and K. Wiesenfeld, Phys. Rev. A 38, 364 (1988).

[14] S.S. Manna, J. Phys. A 24, L363 (1991).

[15] D. Dhar, Studying Self-Organized Criticality with Exactly Solved Models, arXiv:cond-mat/9909009

[16] P. Grassberger and S.S. Manna, J. Phys. (Paris) 51, 1077 (1990). 
[17] S.S. Manna, Physica A 179, 249 (1991).

[18] R. Karmakar, S. S. Manna, and A. L. Stella, cond-mat/0312127

[19] K.-I. Goh, D.-S. Lee, B. Kahng and D. Kim, Phys. Rev. Lett. 91, 148701 (2003).

[20] S. S. Manna and A Kabakcioglu, J. Phys. A, 36, L279 (2003).
[21] A. Vespignani, R. Dickman, M. A. Muñoz and S. Zapperi, Phys. Rev. E. 62, 4564 (2000).

[22] M. De Menech, A. L. Stella, and C. Tebaldi, Phys. Rev. E 58, 2677 (1998).

[23] C. Tebaldi, M. De Menech and A. L. Stella, Phys. Rev. Lett. 83, 3952 (1999). 